\title{
Calculation of Electric Circuits Using the Fast Kirchhoff Method
}

\author{
Viliam Ďuriš ${ }^{1}$, Aleksey G. Chertanovskiy ${ }^{2}$, Sergey G. Chumarov ${ }^{2}$, \\ Aleksander V. Kartuzov ${ }^{3}$ \\ ${ }^{1}$ Department of Mathematics, Constantine The Philosopher University in Nitra, \\ Tr. A. Hlinku 1, 94901, Nitra, Slovakia \\ ${ }^{2}$ Department of Radio Engineering, I. N. Ulyanov Chuvash State University, Moskovsky av., \\ 15, Cheboksary, Russia \\ ${ }^{3}$ Department of Automation and Control in Technical Systems, I. N. Ulyanov Chuvash State University, \\ Moskovsky av., 15, Cheboksary, Russia
}

\begin{abstract}
The Laws of Ohm (1826) and Kirchhoff's laws (1847) are the basis for the theory of electric circuits. Using them when calculating electrical circuits, we need to create and solve a system of equations for circuit currents and nodal voltages. Kirchhoff's Theorem (1847) on finding currents of branches does not require the compilation and solution of this system of equations. Belov G. A. and Zakharov V. G. (2003) re-proved the Kirchhoff's Theorem (1847) and supplemented it with eight rules for calculating electric circuits. With the help of these rules, the currents of branches and nodal voltages are formed from a loop or nodal determinant and an electric circuit. At the same time, duplicate operations occur in the process of forming expressions of currents and voltages according to these rules. In this paper, we prove the theorems of the Fast Kirchhoff method about a complete and truncated tree, about a unique branch and loop, about common branches and loops, as well as the formulas of the Fast Kirchhoff method for generating currents of branches and nodal voltages.
\end{abstract}

DOI: 10.18421/TEM111-09

https://doi.org/10.18421/TEM111-09

Corresponding author: Viliam Ďuriš, Constantine The Philosopher University in Nitra, Tr. A. Hlinku 1, 94901, Nitra, Slovakia

Email: vduris@ukf.sk

Received: 11 September 2021.

Revised: 11 January 2022.

Accepted: 17 January 2022.

Published: 28 February 2022.

(cc)BY-NC-ND (C) 2022 Viliam Ďuriš et al; published by UIKTEN. This work is licensed under the Creative Commons Attribution-NonCommercial-NoDerivs 4.0 License.

The article is published with Open Access at https://www.temjournal.com/
In the Fast Kirchhoff method, when calculating an electric circuit, there are no duplicate operations when generating currents of branches and node voltages, and this reduces the calculation time.

Keywords - Fast Kirchhoff method, proof, electric circuit.

\section{Introduction}

Software packages for modeling electronic circuits showcase a wide range of research. For example they are widely used in modeling nonlinear phenomena [1], in designing electronic circuits [2], and in modeling power electronic devices [3], [4]. These software systems are widely used in education [5], [6], as they allow us to understand what physical processes occur in radio-electronic circuits [7]. Currently, software packages for modeling electronic circuits such as PSpice [8] and NI MultiSim [9], [10] are widely popular. Recently, modeling problems have been solved in close integration with software and hardware [11].

The core of the PSpice and NI MultiSim software packages is the SPICE algorithm [12]. The Foundation of this algorithm is the Laws of Ohm (1826) [13] and Kirchhoff's laws (1847) [14], in which the researchers are still interested [16], [17].

With the help of Kirchhoff's laws, when calculating electrical circuits, we need to create and solve a system of equations for loop currents and nodal voltages. The Theorem of Kirchhoff (1847) [14], on finding the branch current does not require the compilation and solution of this system of equations. Belov G. A. and Zakharov V. G. [15] reproved the Kirchhoff's Theorem and supplemented it with eight rules for calculating electric circuits. Using these rules, the branch currents and the nodal voltages are generated from a loop or base of the determinant and circuits of an electric circuit. Rules of the Kirchhoff-Zakharov method on the basis of resistances [15]: 
1.THE RULE OF CURRENT DUE TO EMF (ELECTROMOTIVE FORCE) SOURCES. The expression for the current in the $j$-th branch due to EMF sources is equal to the fraction

$$
I_{j}^{E}=\frac{\sum \frac{A_{m}}{z_{j}} E_{m}}{\Delta_{z}}
$$

where the denominator is the loop determinant. The numerator is the terms sum consisting of terms of the determinant loop, the cofactor containing resistance $j$-th branch is taken and without this resistance, multiplied by the total EMF of the circuit when removing the remaining resistors in these terms of loop of the determinant. The EMF of branches is taken with a plus sign if their direction coincides with the specified current direction in the $j$-th branch and with a minus sign in the opposite case.

2.THE RULE OF CURRENT DUE TO CURRENT SOURCES. The expression for the current in the $j$-th branch due to current sources is equal to the fraction

$$
I_{j}^{J}=\frac{\sum A_{n} J_{n}}{\Delta_{z}}
$$

where the denominator is the loop determinant. The numerator is the terms sum consisting of terms of the determinant loop, but it does not contain the factor of the resistance $j$-th branch, multiplied by the sum of the currents generated by current sources which belong to the branches with resistances of these components of a loop closing the determinant and using the $j$-th branch in the loops created in the circuit when we remove the resistors included in the factors of these components of the loop of the determinant. Current sources are written in the sum of currents with a plus sign if their direction coincides with the specified current direction in the $j$-th branch and with a minus sign in the opposite case.

3. THE RULE OF THE VOLTAGE BETWEEN THE SPECIFIED AND BASIC NODES, DUE TO EMF SOURCES. The expression for the voltage between the $j$-th and the base node due to EMF sources is equal to the fraction

$$
U_{j}^{E}=\frac{\sum A_{l} E_{l}}{\Delta_{z}}
$$

where the denominator is the loop determinant and the numerator is the sum of summands consisting of the loop determinant summands multiplied by the total EMF of the path from the $j$-th node to the base one, created in the circuit when removing the resistances that are included in the specified loop determinant summands. The EMF of branches is taken with a plus sign if they are directed to the $j$-th node and with a minus sign in the opposite case.
4. THE RULE OF THE VOLTAGE BETWEEN THE SPECIFIED AND BASIC NODES, DUE TO CURRENT SOURCES. The expression for the voltage between the $j$-th and the base node, due to current sources, is equal to the fraction

$$
U_{j}^{J}=\frac{\sum\left(A_{l} \sum z_{r} J_{r}\right)}{\Delta_{z}},
$$

where the denominator is the loop determinant and the numerator is the sum of terms consisting of terms of the loop of the determinant multiplied by the total voltage of the path from the $j$-th node to the base created in the circuit of the remaining resistances and current sources when removing branches with the resistances included the factors listed in terms of the loop of the determinant. The voltages generated at the resistance of the current sources are taken with a plus sign if they are directed to the $j$-th node and negative otherwise.

\section{Theoretical Basis}

We perform a proof of the theorems and formulas of the Fast Kirchhoff method, assuming that the circuit has $n$-branches.

THEOREM 1 (ON THE UNIQUE PATH). If in the circuit, when removing the resistances of a summand, according to rules 3 and 4 (above), a path is created from the branches of a full tree, then this path is the unique one.

PROOF. We remove branches from the circuit with the resistances of the summand of the loop determinant $z_{k}, \ldots, z_{2}, z_{1}$. If in the resulting circuit there forms a path from the $j$-th node to the base with impedance $z_{n}, z_{n-1}, \ldots, z_{k+1}$, then it is unique because of the resistance of the complete tree circuit $z_{n}, z_{n-1}, \ldots, z_{k+1}$, and the resistance summand loop of the determinant $z_{k}, \ldots, z_{2}, z_{1}$ forms a complete set of impedance circuits $z_{n}, z_{n-1}, \ldots, z_{2}, z_{1}$.

THEOREM 2 (ON THE COMMON PATH). If in the circuit, when removing the resistances of a summand, according to rules 3 and 4 (above), a path is created from the branches of an incomplete tree, then this path is common by several terms.

ProOF. Suppose that when we remove the branches with resistances of the summand loop of the determinant $z_{k}, \ldots, z_{2}, z_{1}$ it forms a path from the $j$-th node to the base through the branches of an incomplete tree $\left(z_{n}, z_{n-1}, \ldots, z_{k+m}\right)$, and if for another summand loop of the determinant it will also form a path from the $j$-th node to the base through the same branches of an incomplete tree $\left(z_{n}, z_{n-1}, \ldots, z_{k+m}\right)$, then this path is common to these resistance terms and will change in the range of $z_{k+m}, Z_{k+m-1}, \ldots, Z_{k+m+1}, Z_{k-m}$. 
THEOREM 3 (ON THE UNIQUE LOOP). If in the circuit, when removing the resistances of the summand according to rule 1 (above) a loop is created from the branches of the full tree and the $j$-th branch, then this loop is the unique one.

PROOF. We remove a branch from the circuit with the resistances of the termed loop determinant $z_{k}, \ldots, z_{j+1}, z_{j-1}, \ldots, z_{1}$, taken without the resistance of the $j$-th branch of $z_{j}$. If the circuit is formed, the loop of the $j$-th branch with impedance $z_{j}$ and with branches full of trees with impedance $z_{n}, z_{n-1}, \ldots, z_{k+1}$, then it is unique because of the resistance of the complete tree circuit $z_{n}, z_{n-1}, \ldots, z_{k+1}$, and the resistance summand of the loop of the determinant $z_{k}, \ldots, z_{j+1}, z_{j}, z_{j-1}, \ldots, z_{1}$ forms the full set of impedance circuits $z_{n}, z_{n-1}, \ldots, z_{2}, z_{1}$.

THEOREM 4 (ON THE COMMON LOOP). If in the circuit, when removing the resistances of a summand, according to rule 1 (above), a loop is created from the branches of an incomplete tree and the $j$-th branch, then this loop is common for several terms (stages).

PROOF. Suppose that when we remove branches with the resistances of the summand loop determinant $z_{k}, \ldots, z_{j+1}, z_{j-1}, \ldots, z_{1}$, taken without the resistance of the $j$-th branch $z_{j}$, a loop is formed from the $j$-th branch with resistance $z_{j}$, and the branches of the incomplete tree, with resistances $z_{n}, z_{n-1}, \ldots, z_{k+m}$. If for another summand of the loop the determinant is taken without the resistance of the $j$-th branch of $z_{j}$, a loop will also be formed from the $j$-th branch, with resistance $z_{j}$, and branches of an incomplete tree, with resistances $z_{n}, z_{n-1}, \ldots, z_{k+m}$, then this loop will be common for these terms, and the resistances in the terms will vary within $z_{k+m}, z_{k+m-1}, \ldots, z_{k-m+1}, z_{k-m}$.

THEOREM 5 (ON SUPERPOSITION IN UNIQUE LOOPS). If we remove the resistance summand according to rule 2 (above) and the circuit forms a tree, and if the $j$-th branch through the current source remote branches are formed in the loop, the superposition of these currents in the $j$-th branch is unique.

PROOF. Suppose that when we remove branches from the circuit with the resistances of the summand of the loop determinant $z_{n}, \ldots, z_{l}, \ldots, z_{k}$, a tree is formed in the circuit. If a circuit is formed through the current source $J_{l}$, and the $j$-th branch, and such circuits will be formed for all current sources $J_{n}, J_{n-1}, \ldots, J_{k+1}, J_{k}$, then the superposition of currents in the $j$-th branch is unique, since the other summand of the loop determinant has other combinations of resistances.
THEOREM 6 (ON SUPERPOSITION IN COMMON LOOPS). If in the circuit when removing the resistances of the summand, according to rule 2 (above), a tree is formed, and if the $j$-th branch does not form through all the current sources of the remote branches, then the superposition of these currents in the $j$-th branch will be common for several terms.

PROOF. Suppose that when we remove branches from the circuit with the resistances of the summand of the loop determinant $z_{n}, \ldots, z_{l}, \ldots, z_{k}$, a tree is formed in the circuit. If the circuit through the current source $J_{l}$ is not formed through the $j$-th branch, i.e., the current of the source $J_{l}$ is not superimposed in the $j$-th branch, and there are several such loops, then a combination of common tree branches is possible for several terms, through which currents are superimposed in the $j$-th branch, i.e., the superposition of currents will be the same for several terms of the loop determinant.

FORMULA 1 (CURRENT DUE TO EMF SOURCES). The current in the $j$-th branch due to EMF sources is equal to the fraction

$$
I_{j}^{E}=\frac{\sum_{m=1}^{M} \frac{Z_{m}}{Z_{j}} E_{m}+\sum_{n=M+1}^{N}\left(\sum_{l \cdot n}^{L \cdot n} \frac{Z_{l \cdot n}}{Z_{j}}\right) E_{n}}{\Delta_{z}},
$$

where $Z_{m}$ is the component of a loop of the determinant, in which the resistance of the $j$-th branch includes a co-factor, and after removal of the factors of resistance $j$-th branch in the circuit forms a unique loop; $Z_{l \cdot n}$ is the summand of the loop determinant, in which the resistance of the $j$-th branch includes a co-factor, and after removal of the factors of resistance, $j$-th branch in the circuit is formed in the common loop; $L \cdot n$ is the number of terms of the loop of the determinant, for which the circuit is formed from the same common loop; $M$ is the total number of unique loops; $N$ is the total number of all loops and $E_{m}, E_{n}$ the total EMF of the loop.

PROOF. Under the current rule, due to the sources of EMF [21] while removing from the circuit of factors, the summand from which the resistance of the $j$-th branch is removed, the circuit is formed in this case, the numerator of the expression for the current $j$-th branch is the sum of products summand, the loop of the determinant is taken without resistance by the $j$-th branch and is multiplied by the total EMF of the circuit. From the proof of theorems 3 and 4, the loops created in the circuit can be unique or common. In addition, a common loop is formed for several summands, and these loops will have the same total EMF.

FORMULA 2 (CURRENT DUE TO CURRENT SOURCES). The current of the $j$-th branch due to current sources is equal to the fraction 


$$
I_{j}^{J}=\frac{\sum_{o=1}^{O} Z_{o} J_{o}+\sum_{s=O+1}^{S}\left(A_{s} \sum_{l \cdot s}^{L \cdot S} B_{l \cdot s}\right) J_{s}}{\Delta_{z}},
$$

where $Z_{o}$ is the loop summand of the determinant, for which the circuit is formed from unique superposition currents through the $j$-th branch; $A_{s}$ are the cofactors of the determinant components of the loop for which the circuits are formed, the total superposition of currents through the $j$-th branch; $B_{l \cdot s}$ is the loop of the factors of summands of the determinant, the current sources, which in the circuit does not superimpose a current on the $j$-th branch; $L \cdot S$ is the number of summands of the loop determinant for which there are current sources that do not superimpose their current on the $j$-th branch; $O$ is the the total number of unique superposition currents; $S$ is the total number of all common superposition currents and $J_{o}, J_{S}$ the sum of the loop currents' superposition.

PROOF. Under the current rule, due to the current sources [21] when removing factors of a summand from the circuit, a tree is formed, the numerator of the expression for the current $j$-th branch is the sum of products summand the loop of the determinant on the total current obtained in the circuits through the $j$-th branch and the current sources in the branches of remote resistance. From the proof of theorems 5 and 6 in the circuit, the superposition currents can be unique or common. In this case, the total superposition currents are formed for several summands, for which there will be the same sum of currents.

FoRMULA 3 (VOLTAGE DUE TO EMF SOURCES). The voltage between $j$-th and the base node due to EMF sources is equal to the fraction

$$
U_{j}^{E}=\frac{\sum_{f=1}^{F} Z_{f} E_{f}+\sum_{p=F+1}^{P}\left(\sum_{l \cdot p=1}^{L \cdot p} Z_{l \cdot p}\right) E_{p}}{\Delta_{z}},
$$

where $Z_{f}$ is the loop summand of the determinant of what in the circuit has formed the unique path; $Z_{l \cdot p}$ is the summand of the determinant loop with it, for it is in the circuit formed by the common path; $L \cdot p$ is the number of terms of the loop of the determinant, for which the circuit has formed the same path; $F$ is the total number of unique paths; $P$ is the total number of all paths and $E_{f}, E_{p}$ the path EMF.

PROOF. According to the rule of stress caused by EMF sources [21], when removing the summand multipliers from the circuit, a path is formed. In this case, the numerator of the stress expression between $j$-th and the base node will be the sum of the products of the summands of the loop determinant multiplied by the total EMF of this path. From the proof of theorems 1 and 2, the paths created in the circuit can be unique or common. In this case, a common path is formed for several summands, and these paths will have the same total EMF.
Formula 4 (VOLTAGE DUE TO CURRENT SOURCES). The voltage between $j$-th and the base node due to EMF sources is equal to the fraction

$$
U_{j}^{E}=\frac{\sum_{f=1}^{F} z_{f} \sum_{w \cdot f}^{W \cdot f} z_{w \cdot f} J_{w \cdot f}+\sum_{p=F+1}^{P}\left(\sum_{l \cdot p=1}^{L \cdot p} z_{l \cdot p}\right) \sum_{w \cdot p=1}^{W \cdot p} z_{w \cdot p} J_{w \cdot p}}{\Delta_{Z}}
$$

where $Z_{f}$ is the loop summand of the determinant of what in the circuit has formed the unique path; $Z_{l \cdot p}$ is the summand of the determinant loop with it, for it is in the circuit formed by the common path; $L \cdot p$ is the number of terms of the loop of the determinant, for which the circuit has formed the same path; $F$ is the total number of unique paths; $P$ is the total number of all paths; $z_{w \cdot f}, z_{w \cdot p}$ is the resistance through which a path is formed in the circuit; $J_{w \cdot f}$ is the current source connected parallel to resistance $z_{w \cdot f}$ and $J_{w \cdot p}$ the current source connected parallel to resistance $Z_{w \cdot p}$.

PROOF. under regulation voltage caused by current sources [21], when deleting factors of the summand from the circuit formed in this path, the numerator of the expression of the tension between $j$-th and the reference node will be the sum of products of terms of the loop of the determinant multiplied by the sum of the voltage path, which is equal to the product of the resistance through which the path is created, connected to the current source. From the proof of theorems 1 and 2, the paths created in the circuit can be unique or common. In this case, a common path is formed for several summands, and the total stress for these paths will be the same.

\section{Results}

Let's calculate current $I_{2}$ and voltage $U_{2}$ in the circuit shown in Figure 1.

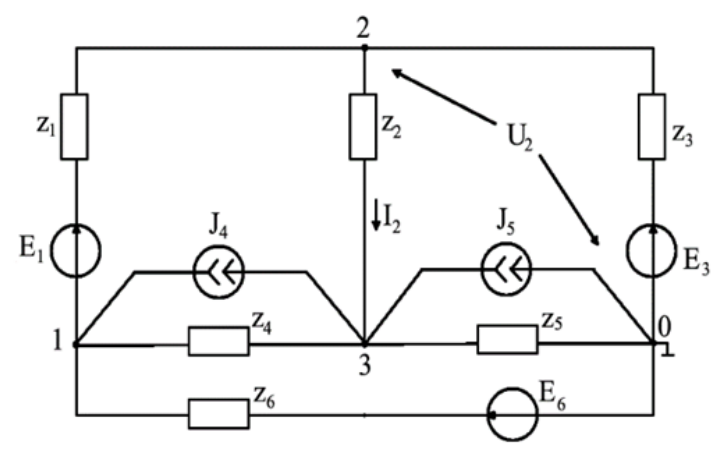

Figure 1. Circuit for calculation current and voltage

The loop determinant of this circuit in Figure 1. is:

$\Delta_{z}=z_{1} z_{2} z_{4}+z_{1} z_{2} z_{5}+z_{1} z_{2} z_{6}+z_{1} z_{3} z_{4}+z_{1} z_{3} z_{5}+$ 


$$
\begin{aligned}
& +z_{1} z_{3} z_{6}+z_{1} z_{4} z_{5}+z_{1} z_{5} z_{6}+z_{2} z_{3} z_{4}+z_{2} z_{3} z_{5}+ \\
& +z_{2} z_{3} z_{6}+z_{2} z_{4} z_{6}+z_{2} z_{5} z_{6}+z_{3} z_{4} z_{5}+z_{3} z_{4} z_{6}+ \\
& +z_{4} z_{5} z_{6} .
\end{aligned}
$$

1. Perform the calculation of current $I_{2}$ due to EMF sources.

Calculation algorithm:

- Select all the summands of the loop determinant for which resistance $z_{2}$ is a factor, and remove resistance $z_{2}: z_{1} z_{4}, z_{1} z_{5}, z_{1} z_{6}, z_{3} z_{4}, z_{3} z_{5}, z_{3} z_{6}$, $z_{4} Z_{6}, Z_{5} Z_{6}$.

- Find all the unique and common loops in the circuit and group all the terms for common loops (Table 1.).

- Find the total EMF for all the found loops (Table 1.).

Table 1. Multipliers for calculating current $i_{2}$ due to EMF sources

\begin{tabular}{|c|c|c|c|}
\hline Summands & Loop & Loop type & Total EMF \\
\hline$z_{1} z_{5}$ & $z_{2} z_{4} z_{6} z_{3}$ & unique & $E_{3}-E_{6}$ \\
\hline$z_{3} z_{4}$ & $z_{2} z_{5} z_{6} z_{1}$ & unique & $E_{1}+E_{6}$ \\
\hline$z_{1} z_{4}, z_{1} z_{6}, z_{4} z_{6}$ & $z_{2} z_{5} z_{3}$ & common & $E_{3}$ \\
\hline$z_{3} z_{5}, z_{3} z_{6}, z_{5} z_{6}$ & $z_{2} z_{4} z_{1}$ & common & $E_{1}$ \\
\hline
\end{tabular}

According to formula (5), current $I_{2}$ due to EMF sources is equal to:

$I_{2}^{E}=$

$\frac{z_{1} z_{5}\left(E_{3}-E_{6}\right)+z_{3} z_{4}\left(E_{1}+E_{6}\right)+\left(z_{1} z_{4}+z_{1} z_{6}+z_{4} z_{6}\right) E_{3}+\left(z_{3} z_{5}+z_{3} z_{6}+z_{5} z_{6}\right) E_{1}}{\Delta_{z}}(10)$

2. Perform the calculation of current $I_{2}$ due to current sources.

Calculation algorithm:

- Select all the summands of the loop determinant for which resistance $z_{2}$ is not a factor:

$$
\begin{gathered}
z_{1} z_{3} z_{4}, z_{1} z_{3} z_{5}, z_{1} z_{3} z_{6}, z_{1} z_{4} z_{5}, z_{1} z_{5} z_{6}, z_{3} z_{4} z_{5}, \\
z_{3} z_{4} z_{6}, z_{4} z_{5} z_{6}
\end{gathered}
$$

- Find all the unique and common superposition loops in the circuit and group all the summands for the common superposition loops (Table 2.).

- Find the total superposition currents (Table 2.).
Table 2. Multipliers for calculating current $i_{2}$ due to current sources

\begin{tabular}{|c|c|c|c|}
\hline Summands & $\begin{array}{c}\text { Loop } \\
\text { superposition } \\
\text { of the current } \\
\text { through the } \\
\text { resistance } z_{2}\end{array}$ & $\begin{array}{c}\text { Current } \\
\text { superposition }\end{array}$ & $\begin{array}{c}\text { Total } \\
\text { current }\end{array}$ \\
\hline$z_{1} z_{5} z_{6}$ & $z_{1}, z_{5}, z_{6}-$ yes & unique & $-J_{5}$ \\
\hline$z_{3} z_{4} z_{6}$ & $z_{3}, z_{4}, z_{6}-$ yes & unique & $J_{4}$ \\
\hline $\begin{array}{c}z_{1} z_{3} z_{4}, \\
z_{1} z_{3} z_{5}, \\
z_{1} z_{3} z_{6}\end{array}$ & $\begin{array}{c}z_{1}, z_{3}-\text { yes } \\
z_{4}, z_{5}, z_{6}-\text { no }\end{array}$ & common & 0 \\
\hline $\begin{array}{c}z_{1} z_{4} z_{5}, \\
z_{3} z_{4} z_{5}, \\
z_{4} z_{5} z_{6}\end{array}$ & $\begin{array}{c}z_{4}, z_{5}-\text { yes } \\
z_{1}, z_{3}, z_{6}-\text { no }\end{array}$ & common & $J_{4}-J_{5}$ \\
\hline
\end{tabular}

According to formula (6), current $I_{2}$ due to the current sources is equal to:

$I_{2}^{J}=\frac{z_{1} z_{5} z_{6}\left(-J_{5}\right)+z_{3} z_{4} z_{6} J_{4}+z_{1} z_{4}\left(z_{4}+z_{5}+z_{6}\right) 0+z_{4} z_{5}\left(z_{1}+z_{3}+z_{6}\right)\left(J_{4}-J_{5}\right)}{\Delta_{z}}$

3. Perform the calculation of voltage $U_{2}$ due to EMF sources.

Calculation algorithm:

- Find all the unique and common paths in the circuit and group all the summands for common paths (Table 3.).

- Find the total EMF for all the paths found (Table 3.).

Table 3. Multipliers for calculating voltage $u_{2}$ due to emf sources

\begin{tabular}{|c|c|c|c|}
\hline Path & Summands & $\begin{array}{c}\text { Path } \\
\text { type }\end{array}$ & $\begin{array}{c}\text { Total } \\
\text { EMF }\end{array}$ \\
\hline$z_{1} z_{4} z_{5}$ & $z_{2} z_{3} z_{6}$ & unique & $E_{1}$ \\
\hline$z_{2} z_{4} z_{6}$ & $z_{1} z_{3} z_{5}$ & unique & $E_{6}$ \\
\hline$z_{2} z_{5}$ & $z_{1} z_{3} z_{4}, z_{1} z_{3} z_{6}, z_{3} z_{4} z_{6}$ & common & 0 \\
\hline$z_{1} z_{6}$ & $z_{2} z_{3} z_{4}, z_{2} z_{3} z_{5}, z_{3} z_{4} z_{5}$ & common & $E_{1}+E_{6}$ \\
\hline$z_{3}$ & $\begin{array}{r}z_{1} z_{2} z_{4}, z_{1} z_{2} z_{5}, z_{1} z_{2} z_{6}, \\
z_{1} z_{4} z_{5}, z_{1} z_{5} z_{6}, z_{2} z_{4} z_{6}, \\
z_{2} z_{5} z_{6}, z_{4} z_{5} z_{6}\end{array}$ & common & $E_{3}$ \\
\hline
\end{tabular}


According to formula (7), voltage $U_{2}$ due to EMF sources is equal to:

$$
\begin{gathered}
U_{2}^{E}=\left[z_{2} z_{3} z_{6} E_{1}+z_{1} z_{3} z_{5} E_{6}+\left(z_{1} z_{3} z_{4}+z_{1} z_{3} z_{6}+z_{3} z_{4} z_{6}\right) 0+\right. \\
+\left(z_{2} z_{3} z_{4}+z_{2} z_{3} z_{5}+z_{3} z_{4} z_{5}\right)\left(E_{1}+E_{6}\right)+
\end{gathered}
$$

$\left.+\left(z_{1} z_{2} z_{4}+z_{1} z_{2} z_{5}+z_{1} z_{2} z_{6}+z_{1} z_{4} z_{5}+z_{1} z_{5} z_{6}+z_{2} z_{4} z_{6}+z_{2} z_{5} z_{6}+z_{4} z_{5} z_{6}\right) E_{3}\right] / \Delta_{z}$

4. Perform the calculation of voltage $U_{2}$ due to current sources.

Calculation algorithm:

- Find all the unique and common paths in the circuit and group all the summands for common paths (Tab. 4.).

- Find the total voltage due to the current sources for all the paths found (Table 4.).

- According to formula (8), voltage $U_{2}$ due to current sources is equal to:

\footnotetext{
$U_{2}^{J}=\left[z_{2} z_{3} z_{6}\left(z_{4} J_{4}+z_{5} J_{5}\right)+\left(z_{1} z_{3} z_{4}+z_{1} z_{3} z_{6}+z_{3} z_{4} z_{6}\right) z_{5} J_{5}+\right.$ $+\left(z_{2} z_{3} z_{4}+z_{2} z_{3} z_{5}+z_{3} z_{4} z_{5}\right) 0+z_{1} z_{3} z_{5}\left(-z_{4} J_{4}\right)+$

$\left.+\left(z_{1} z_{2} z_{4}+z_{1} z_{2} z_{5}+z_{1} z_{2} z_{6}+z_{1} z_{4} z_{5}+z_{1} z_{5} z_{6}+z_{2} z_{4} z_{6}+z_{2} z_{5} z_{6}+z_{4} z_{5} z_{6}\right) 0\right] / \Delta_{z}$
}

Table 4. Multipliers for calculating voltage $U_{2}$ due to current sources

\begin{tabular}{|c|c|c|c|}
\hline Path & Summands & Path type & Total EMF \\
\hline$z_{1} z_{4} z_{5}$ & $z_{2} z_{3} z_{6}$ & unique & $z_{4} J_{4}+z_{5} J_{5}$ \\
\hline$z_{2} z_{4} z_{6}$ & $z_{1} z_{3} z_{5}$ & unique & $-z_{4} J_{4}$ \\
\hline & $z_{1} z_{3} z_{4}$, & & \\
$z_{2} z_{5}$ & $z_{1} z_{3} z_{6}$, & common & $z_{5} J_{5}$ \\
& $z_{3} z_{4} z_{6}$ & & \\
\hline \multirow{5}{*}{$z_{1} z_{6}$} & $z_{2} z_{3} z_{4}$, & & \\
& $z_{2} z_{3} z_{5}$, & common & 0 \\
& $z_{3} z_{4} z_{5}$ & & \\
\hline & $z_{1} z_{2} z_{4}$, & & \\
& $z_{1} z_{2} z_{5}$, & & \\
& $z_{1} z_{2} z_{6}$, & & \\
& $z_{1} z_{4} z_{5}$, & \multirow{2}{*}{ common } & \\
& $z_{1} z_{5} z_{6}$, & & \\
& $z_{2} z_{4} z_{6}$, & & \\
& $z_{2} z_{5} z_{6}$, & & \\
& $z_{4} z_{5} z_{6}$ & & \\
\hline
\end{tabular}

\section{Conclusion}

The paper proves the theorems and formulas of the fast Kirchhoff method on the basis of resistances. Using this method, we can get expressions of currents and voltages in an electric circuit directly from the circuit and the loop determinant. In contrast to methods based on Kirchhoff's laws [17, 18], this approach does not require solving systems of linear equations. In the Fast Kirchhoff method, we do not need to perform duplicate operations, as in the Kirchhoff-Zakharov method for finding the total EMF of the circuit, the total superposition current, the total EMF, or the total path voltage, which significantly reduces the number of operations when calculating currents and voltages in the electrical circuit. This is evident from the calculation of current and voltages in the circuit (Figure 1.):

When calculating the current due to sources of EMF using the Kirchhoff-Zakharov method, requires 8 times to search the sum of all the EMF in the circuit, and in the Fast Kirchhoff method this operation had to be only 4 times and take the components of the loop of the determinant for which the Theorem 1 holds.

When calculating the current due to current sources using the Kirchhoff-Zakharov method, we need to search for the total current superposition 8 times, and in the Fast Kirchhoff method, we need to perform this operation only 4 times and take the summands of the loop determinant for which the Theorem 2 holds.

When calculating the voltage by the KirchhoffZakharov method, it takes 16 times to search for the sum of all EMF or path voltages in the circuit, and in the Fast Kirchhoff method, this operation only needed to be performed 5 times and take the summands of the loop determinant for which the Theorem 3 or the Theorem 4 holds. 


\section{References}

[1]. Aouzale, N., Chitnalah, A., \& Jakjoud, H. (2009, December). PSpice modelling nonlinearity effects on ultrasonic waves. In 2009 International Conference on Microelectronics-ICM (pp. 82-85). IEEE. doi: $10.1109 / \mathrm{icm} .2009 .5418582$.

[2]. Zhao, W., \& Wei, P. (2013, July). PSpice system simulation application in electronic circuit design. In Proceedings of the 32nd Chinese Control Conference (pp. 8634-8636). IEEE.

[3]. Agrawal, A., Gupta, A., Verma, R., \& Jaiswal, A. (2013, February). Simulation of series resonant inverter with unidirectional switches via multisim. In 2013 International Conference on Power, Energy and Control (ICPEC) (pp. 287-289). IEEE.

[4]. Cheng, Z. (2011, July). The application of Multisim in power electronic technology. In 2011 International Conference on Multimedia Technology (pp. 813-816). IEEE.

[5]. Lilyanova, I. (2019, June). Innovation in the teaching methodology of electronics. In 2019 16th Conference on Electrical Machines, Drives and Power Systems (ELMA) (pp. 1-5). IEEE.

[6]. Heying, B., Kejie, D., \& Li, J. (2010, December). Application multisim to virtual laboratory for experiment teaching. In 2010 International Conference on Computational Intelligence and Software Engineering (pp. 1-4). IEEE.

[7]. Makarenko, V., \& Spivak, V. (2016, February). Using circuit simulation to study the processes in electronic circuits. In 2016 13th International Conference on Modern Problems of Radio Engineering, Telecommunications and Computer Science (TCSET) (pp. 76-78). IEEE.

[8]. Tobin, P. (2007). PSpice for circuit theory and electronic devices. Synthesis Lectures On Digital Circuits and Systems, 2(1), 1-159.
[9]. Báez-López, D., \& Guerrero-Castro, F. E. (2011). Circuit analysis with multisim. Synthesis Lectures on Digital Circuits and Systems, 6(3), 1-198.

[10]. Báez-López, D., Guerrero-Castro, F. E., \& Cervantes-Villagómez, O. D. (2012). Advanced circuit simulation using Multisim Workbench. Synthesis Lectures on Digital Circuits and Systems, 7(1), 1-144.

[11]. Hanini, W., \& Ayadi, M. (2017, December). PSpice and Simulink co-simulation for diode and AC-DC converter using SLPS interface software. In 2017 18th international conference on sciences and techniques of automatic control and computer engineering (STA) (pp. 408-413). IEEE.

[12]. eCircuit Center. (2021). Spice algorithm overview. Retrieved from:

http://www.ecircuitcenter.com/SpiceTopics/Overview /Overview.htm [accessed: 05 September 2021].

[13]. Ohm, G. S. (1827). Die galvanische kette: mathematisch. TH Riemann.

[14]. Kirchhoff, G. (1847). Ueber die Auflösung der Gleichungen, auf welche man bei der Untersuchung der linearen Vertheilung galvanischer Ströme geführt wird. Annalen der Physik, 148(12), 497-508.

[15]. G. A. Belov and V. G. Zaharov. (2003). "Application of symbolic circuit functions for calculating linear electrical circuits", Elektrichestvo, 8, 34-41.

[16]. Quintela, F. R., Redondo, R. C., Melchor, N. R., \& Redondo, M. (2009). A general approach to Kirchhoff's Laws. IEEE Transactions on Education, 52(2), 273-278.

[17]. Feldmann, P., \& Rohrer, R. A. (1991). Proof of the number of independent Kirchhoff equations in an electrical circuit. IEEE transactions on circuits and systems, 38(7), 681-684. 